\title{
Hypergravity in five dimensions
}

\author{
Oscar Fuentealba $\oplus^{1,2, *}$ Javier Matulich $\oplus^{2, \dagger}$ and Ricardo Troncoso $\oplus^{1, \$}$ \\ ${ }^{1}$ Centro de Estudios Científicos (CECs), Avenida Arturo Prat 514, Valdivia, Chile \\ ${ }^{2}$ Université Libre de Bruxelles, and International Solvay Institutes, Campus Plaine-CP 231, \\ B-1050 Bruxelles, Belgium
}

(Received 28 November 2019; accepted 20 May 2020; published 2 June 2020)

\begin{abstract}
We show that a spin-5/2 field can be consistently coupled to gravitation without cosmological constant in five-dimensional spacetimes. The fermionic gauge "hypersymmetry" requires the presence of a finite number of additional fields, including a couple of $U(1)$ fields, a spinorial two-form, the dual of the graviton (of mixed $(2,1)$ Young symmetry) and a spin-3 field. The gravitational sector of the action is described by the purely quadratic Gauss-Bonnet term, so that the field equations for the metric are of second order. The local gauge symmetries of the full action principle close, without the need of auxiliary fields, for a suitable extension of the "hyper-Poincaré" algebra. Apart from the Poincaré and spin-3/2 generators, it includes a generator of spin 2 and a $U(1)$ central extension. Noteworthy, the algebra admits an invariant trilinear form, and its generators allow us to precisely accommodate the entire field content within a single connection, so that the hypergravity action can be formulated as a gauge theory described by a ChernSimons form in five dimensions.
\end{abstract}

DOI: 10.1103/PhysRevD.101.124002

\section{INTRODUCTION}

Soon after the advent of supersymmetry, it was natural to wonder about the possible superpartners of the graviton [1]. According to the basic rules of supersymmetry, there are just two possibilities. One of them corresponds to a massless spin-3/2 Rarita-Schwinger field, which can be consistently coupled to gravitation. The full theory is widely known as supergravity $[2,3]$ and it has been extensively explored in different contexts (see e.g., [4-8]). One has barely heard about the remaining possibility, which involves a massless field of spin $5 / 2$, and there is a good reason for that: in four spacetime dimensions the theory is inconsistent $[9,10]$ (in agreement with the nogo theorems for interacting higher spin theories, see e.g., [11-13]). The obstructions to achieve a consistent coupling can be seen as follows. The action for a massless spin- $5 / 2$ field on flat space [14-18] possesses a fermionic gauge symmetry, that is not preserved once the spin- $5 / 2$ field is minimally coupled to gravity $[9,10]$. In few words, the variation of the fermionic action under the fermionic gauge

\footnotetext{
*uentealba@cecs.cl

†jmatulic@ulb.ac.be

troncoso@cecs.cl
}

Published by the American Physical Society under the terms of the Creative Commons Attribution 4.0 International license. Further distribution of this work must maintain attribution to the author(s) and the published article's title, journal citation, and DOI. Funded by SCOAP ${ }^{3}$. symmetry goes like the Riemann tensor, while the variation of the Einstein-Hilbert action becomes proportional to the Einstein tensor, leading no room for cancellation. Besides, the consistency of the fermionic field equation implies the vanishing of the Weyl tensor $[9,19]$, which is certainly too stringent as a condition to be imposed on four-dimensional spacetimes. It has also been shown that neither the addition of cosmological constant nor including nonminimal couplings help in order to circumvent these obstructions $[9,19,20]$ (for related discussions see e.g., [25-27], and references therein). A well-known proposal to surmount these obstacles at least at the cubic level is to turn on the cosmological constant $[28,29]$ and proceed to constructing "formally consistent" classical equations of motion [30-32]. Other approaches to construct consistent interactions of higher spin fields include holographic reconstruction [33-35], light-front approach [36-38] and also examples in $3 D$ [39-49].

In three spacetime dimensions the situation is radically different. Indeed, the Weyl tensor identically vanishes in $D=3$, which allows to express the Riemann tensor in terms of the Ricci tensor and the Ricci scalar. Hence, the aforementioned obstructions to couple a spin-5/2 field to gravitation can be successfully bypassed.

Thus, in the next section we summarize the state of the art of hypergravity in three-dimensional spacetimes, aiming to identify some of its key features that can be extended to higher dimensions. Section III is then devoted to analyze some of the identified structures that can indeed be lifted to higher-dimensional spacetimes, paying special attention in 
a particular nonstandard formulation of supergravity in five dimensions that is endowed with further interesting structures for our purposes. The moral of the aforementioned results allows to formulate certain mild and reasonable hypotheses, so that in Sec. IV we show that, once relying on them, an action for hypergravity in five dimensions can be readily found through the standard Noether procedure. Remarkably, we obtain a local field theory described by a finite number of fields, of spin up to 3 , on standard Riemann-Cartan geometry. The local gauge symmetries of the action principle are also unveiled, and then, we also show that the hypergravity action can be formulated as a gauge theory with standard fiber bundle structure, described by a Chern-Simons form in five dimensions. We conclude with some ending remarks in Sec. V, where we briefly analyze the spectrum of the theory around a braneworld-like background configuration, and also possible extensions of our results to higher odd dimensions.

\section{HYPERGRAVITY IN THREE SPACETIME DIMENSIONS}

The consistent coupling of a spin-5/2 field to general relativity in $D=3$ was achieved by Aragone and Deser [50], who dubbed the theory as "hypergravity." It is the first and one of the simplest theories describing a higher spin field coupled to gravitation. The theory can be conveniently formulated in a local frame, so that the action reads

$$
I=\frac{k}{4 \pi} \int 2 R^{a} e_{a}+i \bar{\psi}_{a} D \psi^{a},
$$

where $e^{a}$ stands for the dreibein, and $R^{a}=d \omega^{a}+$ $\frac{1}{2} \epsilon^{a b c} \omega_{b} \omega_{c}$ for the curvature two-form in terms of the dualized spin connection $\omega^{a}=\frac{1}{2} \epsilon^{a b c} \omega_{b c}$. The irreducible spin-5/2 field $\psi^{a}=\psi_{\mu}^{a} d x^{\mu}$ is assumed to be " $\Gamma$-traceless," i.e., $\Gamma^{a} \psi_{a}=0$, whose Lorentz covariant derivative is given by $D \psi^{a}=d \psi^{a}+\frac{1}{2} \omega^{b} \Gamma_{b} \psi^{a}+\epsilon^{a b c} \omega_{b} \psi_{c}$ [51].

Following the "1.5 formalism" (see, e.g., [4]) it is simple to verify that the theory described by (1) is invariant under

$$
\delta \psi^{a}=D \epsilon^{a} \quad \text { and } \quad \delta e^{a}=\frac{3}{2} i \bar{\epsilon}_{b} \Gamma^{a} \psi^{b},
$$

where the spin-3/2 parameter $\epsilon^{a}=\epsilon^{a}\left(x^{\mu}\right)$ is also $\Gamma$ traceless. In analogy with supergravity [3], the spin connection was proposed to transform as [50]

$$
\delta \omega^{a}=3 i e^{-1} \bar{\epsilon}^{b} \Gamma_{c} f_{b}^{\nu}\left(\frac{1}{2} e_{\nu}^{c} e^{a}-e_{\nu}^{a} e^{c}\right)
$$

with $f_{b}^{\nu}=\varepsilon^{\nu \rho \lambda} D_{\rho} \psi_{\lambda b}$.

In [52], it was shown that hypergravity can be reformulated so that the field content corresponds to the components of a gauge field that takes values in the "hyper-Poincaré" algebra

$$
A=e^{a} P_{a}+\omega^{a} J_{a}+\psi_{a}^{\alpha} Q_{\alpha}^{a},
$$

where the fermionic generators $Q_{\alpha}^{a}$ are $\Gamma$-traceless, and its nonvanishing (anti)commutators read

$$
\begin{aligned}
{\left[J_{a}, J_{b}\right]=} & \varepsilon_{a b c} J^{c}, \quad\left[J_{a}, P_{b}\right]=\varepsilon_{a b c} P^{c}, \\
{\left[J_{a}, Q_{\alpha b}\right]=} & \frac{1}{2}\left(\Gamma_{a}\right)_{\alpha}^{\beta} Q_{\beta b}+\varepsilon_{a b c} Q_{\alpha}^{c}, \\
\left\{Q_{\alpha}^{a}, Q_{\beta}^{b}\right\}= & -\frac{2}{3}\left(C \Gamma^{c}\right)_{\alpha \beta} P_{c} \eta^{a b}+\frac{5}{6} \varepsilon^{a b c} C_{\alpha \beta} P_{c} \\
& +\frac{1}{3}\left(C \Gamma^{(a \mid}\right)_{\alpha \beta} P^{\mid b)} .
\end{aligned}
$$

The hyper-Poincaré algebra (5) admits an invariant bilinear form, whose nonvanishing components are given by

$$
\left\langle J_{a}, P_{b}\right\rangle=\eta_{a b}, \quad\left\langle Q_{\alpha}^{a}, Q_{\beta}^{b}\right\rangle=\frac{2}{3} C_{\alpha \beta} \eta^{a b}-\frac{1}{3} \varepsilon^{a b c}\left(C \Gamma_{c}\right)_{\alpha \beta},
$$

and hence the hypergravity action (1) can be written in terms of a Chern-Simons form

$$
I=\frac{k}{4 \pi} \int\left\langle A d A+\frac{2}{3} A^{3}\right\rangle,
$$

up to a boundary term. The local fermionic hypersymmetry is then spanned by $\delta A=d \lambda+[A, \lambda]$, with $\lambda=\epsilon_{a}^{\alpha} Q_{\alpha}^{a}$, so that

$$
\delta \psi^{a}=D \epsilon^{a}, \quad \delta \omega^{a}=0, \quad \delta e^{a}=\frac{3}{2} i \bar{\epsilon}^{b} \Gamma^{a} \psi_{b},
$$

which agree with (2), (3) only on-shell.

It is worth emphasizing that since the dynamical fields belong to the components of the connection (4), instead of a multiplet, the full set of transformation laws closes in the hyper-Poincaré algebra without the need of auxiliary fields.

Having formulated hypergravity in terms of a ChernSimons action certainly helps in order to unveil the structure of the theory. Indeed, its uniqueness has been recently established by virtue of Becchi-Rouet-StoraTyutin-cohomological techniques [54]. Besides, it was also possible to analyze the asymptotic structure of the theory in [53]. The canonical generators of the asymptotic symmetries were shown to span a hypersymmetric nonlinear extension of the $\mathrm{BMS}_{3}$ algebra (endowed with fermionic generators of conformal weight $5 / 2$ ), with the same central extension as in the bosonic case [55]. This hyper-BMS ${ }_{3}$ algebra was also shown to admit unitary representations [56-58]. Interestingly, as it occurs for $\mathcal{N}=1$ supergravity [59], the anticommutator of the fermionic generators also allows to find (an infinite number of) BPS-like bounds for the bosonic charges, which in the hypersymmetric case turn out to be nonlinear. Circularly symmetric solutions describing both cosmological spacetimes [60-62] 
and conical singularities $[63,64]$ fulfill all of the bounds without saturating them (broken hypersymmetries), while configurations with conical surpluses (angular excess) do not satisfy the bounds. Hypersymmetry bounds saturate for configurations with unbroken hypersymmetries, possessing globally well-defined Killing vector-spinors that fulfill

$$
d \epsilon^{a}+\frac{1}{2} \omega^{b} \Gamma_{b} \epsilon^{a}+\epsilon^{a b c} \omega_{b} \epsilon_{c}=0
$$

In the case of fermions with antiperiodic boundary conditions, the BPS-like configuration that is maximally hypersymmetric corresponds to Minkowski spacetime; while for periodic boundary conditions it is given by the null orbifold [65], possessing a single unbroken hypersymmetry.

It is also worth noting that, as in the case of supergravity $[59,66,67]$, the hypergravity theory can also be extended so as to include parity odd terms in the action [52,53], and consequently, the asymptotic hyper- $\mathrm{BMS}_{3}$ algebra becomes endowed with an additional independent central extension along the Virasoro subalgebra.

Hypergravity in 3D also admits a negative cosmological constant [68-70] and it can be formulated as a ChernSimons theory for $\operatorname{osp}(1 \mid 4) \oplus \operatorname{osp}(1 \mid 4)$, at the expense of including an additional spin-4 field and a Lorentz-like field of mixed symmetry in tangent space. The local Lorentz algebra is then enlarged from $s l(2, R)$ to $s p(4)$, so that the metric is not invariant under spin- 4 gauge symmetries. The asymptotic symmetries are given by the direct sum of two copies of the $W_{\left(2, \frac{5}{2}, 4\right)}$ algebra, from which nonlinear bounds for the bosonic charges can also be established [70,71]. In the limit of vanishing cosmological constant the spin-4 and the Lorentz-like field can be consistently decoupled, so that hypergravity can be suitably formulated in terms of Riemann-Cartan geometry. Furthermore, as shown in [53], the hyper- $\mathrm{BMS}_{3}$ algebra also arises from a suitable truncation of $W_{\left(2, \frac{5}{2}, 4\right)} \oplus W_{\left(2, \frac{5}{2}, 4\right)}$ in the flat limit.

\section{LIFTING TO HIGHER DIMENSIONS}

The formulation of hypergravity in $3 \mathrm{D}$ as a gauge theory of the hyper-Poincaré algebra has been shown to be fruitful, and it is then natural to capture some of its properties that could be lifted to higher dimensions.

In this sense, it is worth highlighting that the hyperPoincaré algebra actually exists for any $D>2$ dimensions [52], whose nonvanishing (anti)commutators read

$$
\begin{aligned}
{\left[J_{a b}, J_{c d}\right] } & =J_{a d} \eta_{b c}-J_{b d} \eta_{a c}+J_{c a} \eta_{b d}-J_{c b} \eta_{a d} \\
{\left[J_{a b}, P_{c}\right] } & =P_{a} \eta_{b c}-P_{b} \eta_{a c} \\
{\left[J_{a b}, Q_{c}^{\alpha}\right] } & =-\frac{1}{2}\left(\Gamma_{a b}\right)_{\beta}^{\alpha} Q_{c}^{\beta}+Q_{a}^{\alpha} \eta_{b c}-Q_{b}^{\alpha} \eta_{a c}
\end{aligned}
$$

$$
\begin{aligned}
{\left[J_{a b}, \bar{Q}_{\alpha c}\right]=} & \frac{1}{2}\left(\Gamma_{a b}\right)^{\beta}{ }_{\alpha} \bar{Q}_{\beta c}+\bar{Q}_{\alpha a} \eta_{b c}-\bar{Q}_{\alpha b} \eta_{a c} \\
\left\{Q^{\alpha a}, \bar{Q}_{\beta}^{b}\right\}= & \frac{3(D-2)}{D^{2}} i\left[(D+1)\left(\Gamma^{c}\right)^{\alpha}{ }_{\beta} P_{c} \eta^{a b}\right. \\
& \left.-\frac{D+2}{D-2}\left(\Gamma^{a b c}\right)^{\alpha}{ }_{\beta} P_{c}-2\left(\Gamma^{(a \mid}\right)^{\alpha}{ }_{\beta} P^{\mid b)}\right]
\end{aligned}
$$

where $\bar{Q}_{a}=Q_{a}^{\dagger} \Gamma^{0}$ generically stands for the Dirac conjugate [73].

Thus, the very existence of the algebra (10), (11) suggests the possibility that hypergravity in higher dimensions might be formulated as a gauge theory of some suitable extension of the hyper-Poincaré group. Indeed, if such extension admitted an invariant tensor of rank $n$, a non-Abelian Chern-Simons action in $D=2 n-1$ dimensions might be a good candidate to explore.

Another interesting lesson that can be extracted from the three-dimensional case without cosmological constant is that, according to the action principle in (1), the spin-5/2 field couples to the geometry exclusively through the spin connection (the dreibein is not involved in the Lorentz covariant derivative). Hence, in our formulation, the fermionic field does not contribute to the stress-energy tensor, but it is noticed by the geometry due to the torsion $T^{a}=\frac{3}{4} i \bar{\psi}_{b} \Gamma^{a} \psi^{b}$. Such type of couplings are certainly excluded in $D=4$ [19], but nonetheless, there is some evidence suggesting that this kind of couplings could be realized in higher odd dimensions. Indeed, a class of supergravity theories featuring fermions that are nonminimally coupled to the curvature, but not to the vielbein, is known to exist for any odd dimensions [74], from which one can also extract a helpful moral.

\section{A. Nonstandard supergravity in five dimensions}

For the sake of simplicity, let us consider the case of (nonstandard) supergravity in five dimensions, whose action reads

$$
I=I_{\mathrm{GB}}-3 I_{b}+I_{\psi}
$$

where

$$
\begin{gathered}
I_{\mathrm{GB}}=\frac{1}{2} \int \varepsilon_{a b c d f} R^{a b} R^{c d} e^{f}, \\
I_{b}=\int R^{a b} R_{a b} b, \\
I_{\psi}=3 \int \bar{\psi} R^{b c} \Gamma_{b c} D \psi+\text { H.c. }
\end{gathered}
$$

Here, the gravitational sector is described by the pure Gauss-Bonnet action $I_{\mathrm{GB}}$ in (13). Since $I_{\mathrm{GB}}$ corresponds to a particular case of Lovelock theory, devoid of the 
Einstein-Hilbert and cosmological terms, the field equations for the metric are of second order.

Invariance under local supersymmetry requires the presence of an additional bosonic term $I_{b}(14)$, where $b=$ $b_{\mu} d x^{\mu}$ stands for an Abelian 1-form.

The dynamics of the spin-3/2 field $\psi=\psi_{\mu} d x^{\mu}$, whose Lorentz covariant derivative is given by $D \psi=d \psi+$ $\frac{1}{4} \omega^{a b} \Gamma_{a b} \psi$, is described by the fermionic term $I_{\psi}$ in (15). Note that the fermionic field nonminimally couples to the spacetime geometry through the curvature two-form $R^{a b}$ (instead of the vielbein).

The action (12) is invariant under the following local supersymmetry transformations

$$
\begin{gathered}
\delta \psi^{a}=D \epsilon^{a}, \quad \delta \omega^{a b}=0, \\
\delta e^{a}=3 i \bar{\epsilon} \Gamma^{a} \psi+\text { H.c. }, \quad \delta b=\bar{\epsilon} \psi+\text { H.c. }
\end{gathered}
$$

It is worth noting that since $I_{\mathrm{GB}}$ in (13) is linear in the vielbein instead of the curvature, the local Lorentz symmetry is extended to the local Poincare group in five dimensions. The full set of local symmetries also includes the gauge transformation associated to the bosonic Abelian field $b$, and hence, their algebra corresponds to superPoincaré with a $U(1)$ central extension, so that the anticommutator of the fermionic generators reads

$$
\left\{Q^{\alpha}, \bar{Q}_{\beta}\right\}=3 i\left(\Gamma^{a}\right)^{\alpha}{ }_{\beta} P_{a}+\delta_{\beta}^{\alpha} K .
$$

The presence of the central extension plays a relevant role, since in this case the super-Poincaré algebra admits an invariant (anti)symmetric form of rank three, whose nonvanishing components are given by

$$
\begin{gathered}
\left\langle J_{a b}, J_{c d}, P_{f}\right\rangle=\frac{2}{3} \varepsilon_{a b c d f}, \\
\left\langle J_{a b}, J_{c d}, K\right\rangle=-4 \eta_{a[c} \eta_{d] b}, \\
\left\langle Q^{\alpha}, J_{a b}, \bar{Q}_{\beta}\right\rangle=-2\left(\Gamma_{a b}\right)_{\beta}^{\alpha},
\end{gathered}
$$

which is crucial in order to formulate this class of supergravity as a gauge theory. Indeed, the field content corresponds to the components of a connection for the centrally extended super-Poincaré algebra

$$
A=e^{a} P_{a}+\frac{1}{2} \omega^{a b} J_{a b}+b K+\bar{\psi} Q-\bar{Q} \psi,
$$

so that the local (super)symmetries are obtained from a gauge transformation $\delta A=d \lambda+[A, \lambda]$, where $\lambda$ takes values on the super-Poincaré algebra with a $U(1)$ central extension. Thus, up to a boundary term, the supergravity action (12) can be written as a Chern-Simons form

$$
I=\int\left\langle A F^{2}-\frac{1}{2} A^{3} F+\frac{1}{10} A^{5}\right\rangle,
$$

where $F=d A+A^{2}$.

In the next section we construct a hypergravity theory in five dimensions that shares some of these features.

\section{HYPERGRAVITY IN FIVE DIMENSIONS}

Following the morals outlined in the previous sections, for a fermionic field $\psi^{a}=\psi_{\mu}^{a} d x^{\mu}$ that fulfills $\Gamma^{a} \psi_{a}=0$, we look for a kinetic term whose coupling to the geometry does not involve the vielbein, being of the form

$$
\bar{\psi}_{a} X^{a b} D \psi_{b}+\text { H.c. }
$$

Local Lorentz invariance then implies that $X^{a b}$ must be necessarily proportional to the curvature two-form $R^{c d}$. It is then simple to verify that there are just five possibilities, so that $X^{a b}$ is given by a combination of the following terms:

$$
\begin{gathered}
R^{a b}, \quad \eta^{a b} R^{c d} \Gamma_{c d} \\
R_{c}^{a} \Gamma^{c b}, \quad \Gamma_{c}^{a} R^{c b}, \quad \epsilon^{a b c d e} R_{c d} \Gamma_{e}
\end{gathered}
$$

Nonetheless, since the fermionic field is $\Gamma$-traceless, the last three possibilities in (25) become redundant, and the relevant ones then reduce to the remaining two in (24). Hence, $X^{a b}$ must be of the form

$$
X^{a}{ }_{b}=\delta^{a}{ }_{b} R^{c d} \Gamma_{c d}+\alpha R^{a}{ }_{b} .
$$

We also assume that the local fermionic gauge symmetry (hypersymmetry) is spanned by a spin-3/2 parameter $\epsilon^{a}$ subject to the $\Gamma$-traceless condition $\left(\Gamma^{a} \epsilon_{a}=0\right)$, so that the transformation law of the fermionic field and the spin connection agree with those in the three-dimensional case, i.e.,

$$
\delta \psi^{a}=D \epsilon^{a}, \quad \delta \omega^{a b}=0 .
$$

As explained below, it is useful to fix the arbitrary constant in $X_{b}^{a}$ according to $\alpha=-4$, since it minimizes the number of additional bosonic fields, and in particular, it avoids the introduction of extra fields with mixed symmetry on tangent space.

\section{A. Action principle and local (hyper)symmetries}

According to the hypotheses outlined at the beginning of this section, the searched for action principle for hypergravity can then be readily obtained through the standard Noether procedure, starting from the fermionic kinetic term in (23), with $X^{a}{ }_{b}$ given by (26) and $\alpha=-4$, and the local hypersymmetry transformation laws in (27). 
The action is then given by

$$
I=I_{\mathrm{GB}}+\frac{9}{5} I_{b}+I_{e^{a b}}+I_{\psi^{a}}
$$

where $I_{\mathrm{GB}}$ stands for the pure Gauss-Bonnet action in (13), $I_{b}$ is given by (14), and the remaining terms read

$$
\begin{gathered}
I_{e^{a b}}=-24 \int R^{a}{ }_{c} R^{c b} e_{a b}, \\
I_{\psi^{a}}=3 \int \bar{\psi}_{a}\left(\delta_{b}^{a} R^{c d} \Gamma_{c d}-4 R_{b}^{a}\right) D \psi^{b}+\text { H.c. }
\end{gathered}
$$

Apart from the vielbein $e^{a}$, the spin connection $\omega^{a b}$ and the fermionic one-form field $\psi^{a}$, the theory includes additional bosonic fields described by an Abelian field $b=b_{\mu} d x^{\mu}$, and a one-form given by $e^{a b}=e_{\mu}^{a b} d x^{\mu}$, being symmetric and traceless in tangent space $\left(e^{a b}=e^{b a}, \eta_{a b} e^{a b}=0\right)$.

The "off-shell" spin can be readily identified through looking at the irreducible components of the fields under the pullback of the local Lorentz group. The fermionic oneform contains a spin-5/2 field $\psi_{(\mu \nu)}$ and a spinorial twoform $\psi_{[\mu \nu]}$, which are symmetric and antisymmetric under $\mu \leftrightarrow \nu$, respectively [75]. Analogously, the one-form $e^{a b}$ decomposes as

$$
e_{\mu}^{a b}:\left\{e_{(\mu \nu \lambda)}, e_{\nu \mu}^{\nu}, e_{\mu \nu, \lambda}\right\},
$$

so that its trace $e^{\nu}{ }_{\nu \mu}$ corresponds to a spin-1 field, while the fully symmetric part $e_{(\mu \nu \lambda)}$ describes the highest spin field $(s=3)$. The remaining field $e_{\mu \nu, \lambda}$ is of mixed $(2,1)$ Young symmetry, remarkably coinciding with the dual of the graviton in five dimensions [77] (see also [78]). It is worth highlighting that in our context, the field $e_{\mu \nu, \lambda}$ is strictly required by hypersymmetry, instead of duality at the linearized level.

We should also emphasize one of the advantages of keeping all of the irreducible components of the fields aforementioned. Indeed, if one wanted to project them out, one might follow the nowadays standard procedure of enlarging the Lorentz group by introducing additional fields of mixed symmetry in tangent space (see e.g., $[18,25,40,76,79,80])$. However, when the spacetime geometry becomes dynamical, the price to pay would be that the metric transforms in a nontrivial way under higher spin transformations, implying that Riemannian geometry should be extended in some suitable way, which is hitherto unknown. Hence, keeping all of the irreducible components aforementioned, allows us to formulate a consistent theory of hypergravity in five dimensions in a conservative way, described by a local field theory with a finite number of fields (of spin up to three) on standard Riemann-Cartan geometry.
The action is invariant under the following local hypersymmetry transformations

$$
\begin{gathered}
\delta \psi^{a}=D \epsilon^{a}, \quad \delta \omega^{a b}=0 . \\
\delta e^{a}=3 i \bar{\epsilon}^{b} \Gamma^{a} \psi_{b}+\text { H.c. } \\
\delta b=\bar{\epsilon}^{a} \psi_{a}+\text { H.c. } \\
\delta e^{a b}=\bar{\epsilon}^{(a} \psi^{b)}-\frac{1}{5} \eta^{a b} \bar{\epsilon}_{c} \psi^{c}+\text { H.c. }
\end{gathered}
$$

Note that the transformations in (32), (33) agree with those in the three-dimensional case (extended for Dirac fermions).

Apart from the manifest local Lorentz invariance, the action (28) is also invariant under local translations, and $U(1)$ gauge transformations with parameters $\lambda^{a}$ and $\lambda$, respectively. An additional local bosonic "spin 1-2-3" symmetry is spanned by a parameter $\lambda^{a b}$, that is symmetric and traceless. The transformation laws of the fields under these symmetries are given by

$$
\begin{gathered}
\delta e^{a}=D \lambda^{a}, \quad \delta b=d \lambda, \quad \delta e^{a b}=D \lambda^{a b}, \\
\delta \omega^{a b}=0, \quad \delta \psi^{a}=0 .
\end{gathered}
$$

The whole set of local gauge symmetries of the hypergravity action (28) turns out to close for an extension of the hyper-Poincaré algebra in (10), (11) in five dimensions, endowed with an additional $U(1)$ generator $K$ and a symmetric and traceless spin-2 generator $P_{a b}$, whose nonvanishing (anti)commutators manifestly read

$$
\begin{aligned}
{\left[J_{a b}, J_{c d}\right]=} & J_{a d} \eta_{b c}-J_{b d} \eta_{a c}+J_{c a} \eta_{b d}-J_{c b} \eta_{a d}, \\
{\left[J_{a b}, P_{c}\right]=} & P_{a} \eta_{b c}-P_{b} \eta_{a c}, \\
{\left[J_{a b}, P_{c d}\right]=} & P_{a d} \eta_{b c}-P_{b d} \eta_{a c}+P_{c a} \eta_{b d}-P_{c b} \eta_{a d}, \\
{\left[J_{a b}, Q_{c}^{\alpha}\right]=} & -\frac{1}{2}\left(\Gamma_{a b}\right)^{\alpha}{ }_{\beta} Q_{c}^{\beta}+Q_{a}^{\alpha} \eta_{b c}-Q_{b}^{\alpha} \eta_{a c}, \\
{\left[J_{a b}, \bar{Q}_{\alpha c}\right]=} & \frac{1}{2}\left(\Gamma_{a b}\right)_{\alpha}^{\beta} \bar{Q}_{\beta c}+\bar{Q}_{\alpha a} \eta_{b c}-\bar{Q}_{\alpha b} \eta_{a c}, \\
\left\{Q^{\alpha a}, \bar{Q}_{\beta}^{b}\right\}= & \frac{54}{25} i\left(\Gamma^{c}\right)_{\beta}^{\alpha} P_{c} \eta^{a b}-\frac{21}{25} i\left(\Gamma^{a b c}\right)_{\beta}^{\alpha} P_{c} \\
& -\frac{18}{25} i\left(\Gamma^{(a \mid}\right)^{\alpha}{ }_{\beta} P^{\mid b)}+\frac{1}{5}\left(4 \eta^{a b} \delta_{\beta}^{\alpha}-\left(\Gamma^{a b}\right)_{\beta}^{\alpha}\right) K \\
& +\frac{1}{10}\left(3 \delta_{\beta}^{\alpha} P^{a b}+2\left(\Gamma_{c}{ }^{[a]}\right)^{\alpha}{ }_{\beta} P^{\mid b] c}\right) .
\end{aligned}
$$

\section{B. Hypergravity as a gauge theory for the extended hyper-Poincaré algebra}

Interestingly, the local gauge symmetries of the hypergravity action close according to the extended 
hyper-Poincaré algebra in (38) without the need of auxiliary fields. Indeed, this is a consequence of the fact that the field content precisely corresponds to the components of a single connection of the extended hyper-Poincaré algebra

$A=e^{a} P_{a}+\frac{1}{2} \omega^{a b} J_{a b}+b K+\frac{1}{2} e^{a b} P_{a b}+\bar{\psi}^{a} Q_{a}-\bar{Q}_{a} \psi^{a}$,

and hence, the local gauge symmetries are recovered from $\delta A=d \eta+[A, \eta]$, where $\eta$ stands for an extended hyperPoincaré-algebra-valued zero-form

$\eta=\lambda^{a} P_{a}+\frac{1}{2} \sigma^{a b} J_{a b}+\lambda K+\frac{1}{2} \lambda^{a b} P_{a b}+\bar{\epsilon}^{a} Q_{a}-\bar{Q}_{a} \epsilon^{a}$.

Besides, the additional bosonic generators $K$ and $P_{a b}$ extend the hyper-Poincare algebra so that it admits an invariant (anti-)symmetric form of rank three. Their nonvanishing components are given by

$$
\begin{aligned}
\left\langle J_{a b}, J_{c d}, P_{f}\right\rangle & =\frac{2}{3} \varepsilon_{a b c d f}, \\
\left\langle J_{a b}, J_{c d}, K\right\rangle & =\frac{12}{5} \eta_{a[c} \eta_{d] b}, \\
\left\langle J_{a b}, J_{c d}, P_{e f}\right\rangle & =-64\left(\eta_{e[a} \eta_{b][c} \eta_{d] f}-\frac{1}{5} \eta_{c[a} \eta_{b] d} \eta_{e f}\right), \\
\left\langle Q_{c}^{\alpha}, J_{a b}, \bar{Q}_{\beta d}\right\rangle & =2\left[4 \mathbb{P}_{c[a} \mathbb{P}_{b] d}-\mathbb{P}_{c e} \Gamma_{a b} \mathbb{P}_{d}^{e}\right]_{\beta}^{\alpha},
\end{aligned}
$$

where the $\Gamma$-traceless projector $\left(\mathbb{P}_{a b}\right)_{\beta}^{\alpha}=\eta_{a b} \delta^{\alpha}{ }_{\beta}-$ $\frac{1}{5}\left(\Gamma_{a} \Gamma_{b}\right)_{\beta}^{\alpha}$, ensures $\Gamma$-traceless of the fermionic entries of the bracket.

Therefore, the hypergravity theory (28) can be formulated as a gauge theory with standard fiber bundle structure. Indeed, by virtue of (39) and (41), the action (28) can be written in terms of a Chern-Simons form in five dimensions

$$
I=\int\left\langle A F^{2}-\frac{1}{2} A^{3} F+\frac{1}{10} A^{5}\right\rangle
$$

up to a boundary term. Note that the field equations can then be compactly expressed in a manifestly covariant way under the extended hyper-Poincaré algebra, spanned by the set $G_{I}=\left\{J_{a b}, P_{a}, K, P_{a b}, Q_{a}, \bar{Q}_{a}\right\}$, according to

$$
\left\langle F^{2} G_{I}\right\rangle=0
$$

Here, $F=d A+A^{2}$ stands for the field strength, whose components are given by

$$
\begin{aligned}
F= & \tilde{T}^{a} P_{a}+\frac{1}{2} R^{a b} J_{a b}+\tilde{T} K+\frac{1}{2} \tilde{T}^{a b} P_{a b} \\
& +D \bar{\psi}^{a} Q_{a}-\bar{Q}_{a} D \psi^{a}
\end{aligned}
$$

where

$$
\begin{gathered}
\tilde{T}^{a}=T^{a}-3 i \bar{\psi}_{b} \Gamma^{a} \psi^{b}, \\
\tilde{T}=d b-\bar{\psi}_{a} \psi^{a}, \\
\tilde{T}^{a b}=D e^{a b}-\bar{\psi}^{(a} \psi^{b)}+\frac{1}{5} \eta^{a b} \bar{\psi}_{c} \psi^{c},
\end{gathered}
$$

and $T^{a}=D e^{a}$ is the torsion.

\section{ENDING REMARKS}

We have proposed an action principle for hypergravity in five-dimensional spacetimes, that can be formulated as a field theory with standard fiber bundle structure. Noteworthy, the theory contains a finite number of higher spin fields (up to just $s=3$ ), that can be seen as the components of a connection for the extended hyperPoincare group. Thus, as the fields are not arranged within the components of an irreducible multiplet, the possible inconsistencies associated to the Haag-ŁopuszańskiSohnius theorem [81] can be successfully circumvented. In particular, bosonic and fermionic degrees of freedom are then not restricted to match.

The theory possesses a simple geometric structure, but as it is the case of generic non-Abelian Chern-Simons theories in five dimensions, the analysis of its dynamics is not so straightforward [82-86], since the constraint structure changes along phase space. A simple way of visualizing this is the following. The field equations (43) are trivially solved by Minkowski spacetime in vacuum, because in that case the field strength $F$ in (44) vanishes. Nevertheless, since the field equations are purely quadratic in $F$, linear perturbations around this (maximally hypersymmetric) configuration possess a "linearization instability," implying that the analysis necessarily requires to go to higher order. As pointed out in $[87,88]$, this feature appears to be welcome, since the theory naturally tends to explore different vacua. Note that solutions in vacuum, without torsion, fulfill the analogue of the Einstein equation

$$
\varepsilon_{a b c d f} R^{a b} R^{c d}=0,
$$

with an additional condition on the geometry that comes from the variation of the action (28) with respect to the bosonic fields $b$ and $e_{a b}$, given by

$$
R_{c}^{a} R^{c b}=0 .
$$

Equations (48) and (49) also emerge from the consistency of the fermionic field equations. It is then worth 
highlighting that the latter condition is not as stringent as requiring the vanishing of the Weyl tensor. Indeed, it is simple to verify that the spherically symmetric solution of (48) (see $[89,90]$ ) automatically fulfills (49), without imposing any additional condition on the geometry.

The field equations (48) and (49) can also be seen to admit braneworld-like solutions in vacuum, whose metric is given by [87]

$$
d s^{2}=e^{-2 a|z|}\left(d z^{2}+\tilde{g}_{\mu \nu}(x) d x^{\mu} d x^{\nu}\right),
$$

where $\tilde{g}_{\mu \nu}$ stands for the metric of a maximally symmetric spacetime along the four-dimensional brane. Remarkably, a precise jump in the extrinsic curvature is allowed by the theory in vacuum, i.e., without the need of an induced stress-energy tensor on the brane. This effect can also be seen to arise from the fact that the analog of the Israel junction conditions in this case become quadratic in the extrinsic curvature, and hence, they admit nontrivial solutions even in vacuum (see e.g., [91]). As in [87], metric perturbations along the brane turn out to possess a welldefined propagator, precisely given by that of a FierzPauli massless graviton, provided that the induced cosmological constant on the braneworld is strictly positive. In other words, perturbations of the metric along the brane reproduce linearized general relativity around a fourdimensional de Sitter spacetime with curvature radius given by $a^{-2}$.

It is also worth pointing out that perturbations of the fermionic fields on the braneworld-like metric (50) appear to reproduce a sort of partially massless version of the Sorokin-Vasiliev doublet [26] (see also [92-94]), on de Sitter spacetime [95].

It would also be interesting to study new dimensional reduction schemes that apply for the class of theories under discussion, as those recently proposed in [96].

Formulating hypergravity in presence of cosmological constant would also be worth to explore. Nonetheless, as in the three-dimensional case [68-70], the introduction of additional bosonic higher spin fields seems to be inevitable. Indeed, different couplings of higher spin fields to gravitation on $\mathrm{AdS}_{5}$ along these lines have also been proposed in [97-99]. It might also be of interest to see whether any of the higher spin algebras already available in the literature could lead to sensible theories in higher odd dimensions.

Besides, the coupling of fermionic fields of half-integer spin with gravitation in three-dimensional spacetimes is also known to be consistent [50], and it has been shown that the theory possesses a suitable reformulation in terms of the hyper-Poincaré algebra with fermionic generators of spin $s=n+\frac{1}{2}[52,100]$. Lifting these results to higher (odd) dimensions then certainly deserves consideration. Indeed, for fermionic generators of spin $s=3 / 2$, preliminary results suggest that hypergravity theories with a finite number of bosonic fields can actually be formulated in odd spacetime dimensions. Interestingly, as in the fivedimensional case, the bosonic field with the highest spin is also given by $s=3$ in $D=5 \bmod 4$ dimensions; while for $D=7 \bmod 4$, no bosonic higher spin fields are required $(s \leq 2)$ [105].

\section{ACKNOWLEDGMENTS}

We thank Konstantin Alkalaev, Nicolas Boulanger, Andrea Campoleoni, Marcela Cárdenas, Hernán González, Eduardo Guendelman, Daniel Grumiller, Marc Henneaux, Miguel Pino, Evgeny Skvortsov, Dmitri Sorokin, David Tempo and Stefan Theisen for helpful discussions. We especially thank Dario Francia and Rakib Rahman for pointing out the relevance of the "hook" fermionic field in the analysis. We also thank the organizers of the ESI Programme and Workshop "Higher Spin and Holography" hosted by the Erwin Schrödinger Institute (ESI), during March in Vienna, for the opportunity of presenting this work. The research of O. F. was partially supported by a Marina Solvay Fellowship. The work of O. F. and J. M. was supported by the ERC Advanced Grant "High-Spin-Grav" and by FNRS-Belgium (convention FRFC PDR T.1025.14 and convention IISN 4.4503.15). This research has been partially supported by Fondecyt Grants No. 1161311, No. 1171162, No. 1181031, No. 1181496, No. 3170772. The Centro de Estudios Científicos (CECs) is funded by the Chilean Government through the Centers of Excellence Base Financing Program of Conicyt.
[1] A. Salam and J. A. Strathdee, On superfields and FermiBose symmetry, Phys. Rev. D 11, 1521 (1975).

[2] D. Z. Freedman, P. van Nieuwenhuizen, and S. Ferrara, Progress toward a theory of supergravity, Phys. Rev. D 13, 3214 (1976).

[3] S. Deser and B. Zumino, Consistent supergravity, Phys. Lett. B 62, 335 (1976).
[4] P. van Nieuwenhuizen, Supergravity, Phys. Rep. 68, 189 (1981).

[5] H. P. Nilles, Supersymmetry, supergravity and particle physics, Phys. Rep. 110, 1 (1984).

[6] H. E. Haber and G. L. Kane, The search for supersymmetry: Probing physics beyond the standard model, Phys. Rep. 117, 75 (1985). 
[7] S. P. Martin, A Supersymmetry primer, Adv. Ser. Dir. High Energy Phys. 18, 1 (1998); 21, 1 (2010).

[8] D.Z. Freedman and A. Van Proeyen, Supergravity (Cambridge University Press, Cambridge, England, 2012).

[9] C. Aragone and S. Deser, Consistency problems of hypergravity, Phys. Lett. 86B, 161 (1979).

[10] F. A. Berends, J. W. van Holten, B. de Wit, and P. van Nieuwenhuizen, On spin 5/2 gauge fields, J. Phys. A 13, 1643 (1980).

[11] X. Bekaert, N. Boulanger, and P. Sundell, How higher-spin gravity surpasses the spin two barrier: No-go theorems versus yes-go examples, Rev. Mod. Phys. 84, 987 (2012).

[12] V. E. Didenko and E. D. Skvortsov, Elements of Vasiliev theory, arXiv:1401.2975.

[13] R. Rahman and M. Taronna, From higher spins to strings: A primer, arXiv:1512.07932.

[14] J. Schwinger, Particles, Sources, and Fields (AddisonWesley, Reading, MA, 1970).

[15] J. Fang and C. Fronsdal, Massless fields with half integral spin, Phys. Rev. D 18, 3630 (1978).

[16] F. A. Berends, J. W. van Holten, P. van Nieuwenhuizen, and B. de Wit, On spin 5/2 gauge fields, Phys. Lett. 83B, 188 (1979); Erratum, Phys. Lett. 84B, 529 (1979).

[17] T. Curtright, Massless field supermultiplets with arbitrary spin, Phys. Lett. 85B, 219 (1979).

[18] M. A. Vasiliev, 'Gauge' form of description of massless fields with arbitrary spin. (in Russian), Yad. Fiz. 32, 855 (1980) [Sov. J. Nucl. Phys. 32, 439 (1980)].

[19] C. Aragone and S. Deser, Higher spin vierbein gauge fermions and hypergravities, Nucl. Phys. B170, 329 (1980).

[20] Similar obstructions also occur for higher rank representations of fields with spin $s \leq 2$. In particular, in the case of a massless spinorial two-form (see e.g., [21,22]), fermionic gauge symmetries are also spoiled when the field is coupled to gravitation [23,24].

[21] P. K. Townsend, Gauge invariance for spin 1/2, Phys. Lett. 90B, 275 (1980).

[22] M. Henneaux, V. Lekeu, and A. Leonard, The action of the (free) (4, 0)-theory, J. High Energy Phys. 01 (2018) 114; Erratum, J. High Energy Phys. 05 (2018) 105.

[23] S. Deser and E. Witten, Dynamical properties of antisymmetric tensor fields, Nucl. Phys. B178, 491 (1981).

[24] S. Deser, P. K. Townsend, and W. Siegel, Higher rank representations of lower spin, Nucl. Phys. B184, 333 (1981).

[25] M. A. Vasiliev, Free massless fields of arbitrary spin in the de Sitter space and initial data for a higher spin superalgebra, Yad. Fiz. 45, 1784 (1987) [Fortsch. Phys. 35, 741 (1987)].

[26] D. P. Sorokin and M. A. Vasiliev, Reducible higher-spin multiplets in flat and AdS spaces and their geometric frame-like formulation, Nucl. Phys. B809, 110 (2009).

[27] K. B. Alkalaev and M. A. Vasiliev, $N=1$ supersymmetric theory of higher spin gauge fields in $\operatorname{AdS}(5)$ at the cubic level, Nucl. Phys. B655, 57 (2003).

[28] E. Fradkin and M.A. Vasiliev, On the gravitational interaction of massless higher spin fields, Phys. Lett. B 189, 89 (1987).
[29] E. Fradkin and M. A. Vasiliev, Cubic interaction in extended theories of massless higher spin fields, Nucl. Phys. B291, 141 (1987).

[30] M. A. Vasiliev, Consistent equation for interacting gauge fields of all spins in $(3+1)$-dimensions, Phys. Lett. B 243, 378 (1990).

[31] M. A. Vasiliev, Higher spin gauge theories: Star product and AdS space, arXiv:hep-th/9910096.

[32] M. Vasiliev, Nonlinear equations for symmetric massless higher spin fields in (A)dS(d), Phys. Lett. B 567, 139 (2003).

[33] X. Bekaert, J. Erdmenger, D. Ponomarev, and C. Sleight, Quartic AdS interactions in higher-spin gravity from conformal field theory, J. High Energy Phys. 11 (2015) 149.

[34] C. Sleight and M. Taronna, Higher Spin Interactions from Conformal Field Theory: The Complete Cubic Couplings, Phys. Rev. Lett. 116, 181602 (2016).

[35] C. Sleight and M. Taronna, Higher-Spin Gauge Theories and Bulk Locality, Phys. Rev. Lett. 121, 171604 (2018).

[36] D. Ponomarev and E. D. Skvortsov, Light-front higherspin theories in flat space, J. Phys. A 50, 095401 (2017).

[37] E. D. Skvortsov, T. Tran, and M. Tsulaia, Quantum Chiral Higher Spin Gravity, Phys. Rev. Lett. 121, 031601 (2018).

[38] E. Skvortsov, Light-front bootstrap for chern-simons matter theories, J. High Energy Phys. 06 (2019) 058.

[39] M. Blencowe, A consistent interacting massless higher spin field theory in $d=(2+1)$, Classical Quantum Gravity 6, 443 (1989).

[40] A. Campoleoni, S. Fredenhagen, S. Pfenninger, and S. Theisen, Asymptotic symmetries of three-dimensional gravity coupled to higher-spin fields, J. High Energy Phys. 11 (2010) 007.

[41] M. Henneaux and S.-J. Rey, Nonlinear $W_{\text {infinity }}$ as asymptotic symmetry of three-dimensional higher spin anti-de Sitter gravity, J. High Energy Phys. 12 (2010) 007.

[42] H. Afshar, A. Bagchi, R. Fareghbal, D. Grumiller, and J. Rosseel, Higher Spin Theory in 3-Dimensional Flat Space, Phys. Rev. Lett. 111, 121603 (2013).

[43] H. A. Gonzalez, J. Matulich, M. Pino, and R. Troncoso, Asymptotically flat spacetimes in three-dimensional higher spin gravity, J. High Energy Phys. 09 (2013) 016.

[44] M. Henneaux, A. Perez, D. Tempo, and R. Troncoso, Chemical potentials in three-dimensional higher spin anti-de Sitter gravity, J. High Energy Phys. 12 (2013) 048.

[45] C. Bunster, M. Henneaux, A. Perez, D. Tempo, and R. Troncoso, Generalized black holes in three-dimensional spacetime, J. High Energy Phys. 05 (2014) 031.

[46] M. Gary, D. Grumiller, M. Riegler, and J. Rosseel, Flat space (higher spin) gravity with chemical potentials, J. High Energy Phys. 01 (2015) 152.

[47] J. Matulich, A. Perez, D. Tempo, and R. Troncoso, Higher spin extension of cosmological spacetimes in 3D: Asymptotically flat behaviour with chemical potentials and thermodynamics, J. High Energy Phys. 05 (2015) 025 .

[48] D. Grumiller, A. Perez, S. Prohazka, D. Tempo, and R. Troncoso, Higher spin black holes with soft hair, J. High Energy Phys. 10 (2016) 119. 
[49] M. Grigoriev, I. Lovrekovic, and E. Skvortsov, New conformal higher spin gravities in $3 d$, J. High Energy Phys. 01 (2020) 059.

[50] C. Aragone and S. Deser, Hypersymmetry in $D=3$ of coupled gravity massless spin 5/2 system, Classical Quantum Gravity 1, L9 (1984).

[51] Wedge product between forms is assumed. We choose the orientation so that the Levi-Civita symbol fulfills $\varepsilon_{012}=1$, and the Minkowski metric $\eta_{a b}$ follows the mostly plus convention. In terms of the charge conjugation matrix $C$, the Majorana conjugate reads $\bar{\psi}_{a \alpha}=\psi_{a}^{\beta} C_{\beta \alpha}$. An imaginary unit " $i$ " in the product of real Grassmann variables goes by hand with $\left(\theta_{1} \theta_{2}\right)^{*}=-\theta_{1} \theta_{2}$. Our convention for (anti) symmetrization differs from that in [52,53]. Afterwards, round and square brackets stand for symmetrization and antisymmetrization of the enclosed indices, respectively, including a $1 / 2$ factor, i.e., $X^{(a \mid} Y^{b} Z^{\mid c)}=\frac{1}{2}\left(X^{a} Y^{b} Z^{c}+\right.$ $\left.X^{c} Y^{b} Z^{a}\right)$, and $X^{[a \mid} Y^{b} Z^{\mid c]}=\frac{1}{2}\left(X^{a} Y^{b} Z^{c}-X^{c} Y^{b} Z^{a}\right)$. The three-dimensional Dirac matrices $\Gamma^{a}$ satisfy the Clifford algebra $\left\{\Gamma_{a}, \Gamma_{b}\right\}=2 \eta_{a b}$.

[52] O. Fuentealba, J. Matulich, and R. Troncoso, Extension of the Poincaré group with half-integer spin generators: Hypergravity and beyond, J. High Energy Phys. 09 (2015) 003.

[53] O. Fuentealba, J. Matulich, and R. Troncoso, Asymptotically flat structure of hypergravity in three spacetime dimensions, J. High Energy Phys. 10 (2015) 009.

[54] R. Rahman, The uniqueness of hypergravity, J. High Energy Phys. 11 (2019) 115.

[55] G. Barnich and G. Compère, Classical central extension for asymptotic symmetries at null infinity in three spacetime dimensions, Classical Quantum Gravity 24, F15 (2007).

[56] A. Campoleoni, H. A. Gonzalez, B. Oblak, and M. Riegler, Rotating higher spin partition functions and extended BMS symmetries, J. High Energy Phys. 04 (2016) 034.

[57] A. Campoleoni, H. A. Gonzalez, B. Oblak, and M. Riegler, BMS modules in three dimensions, Int. J. Mod. Phys. A 31, 1650068 (2016).

[58] B. Oblak, BMS particles in three dimensions, Ph.D. thesis, Brussels U., 2016.

[59] G. Barnich, L. Donnay, J. Matulich, and R. Troncoso, Asymptotic symmetries and dynamics of threedimensional flat supergravity, J. High Energy Phys. 08 (2014) 071.

[60] K. Ezawa, Transition amplitude in $(2+1)$-dimensional Chern-Simons gravity on a torus, Int. J. Mod. Phys. A 09, 4727 (1994).

[61] L. Cornalba and M. S. Costa, A new cosmological scenario in string theory, Phys. Rev. D 66, 066001 (2002).

[62] L. Cornalba and M. S. Costa, Time dependent orbifolds and string cosmology, Fortsch. Phys. 52, 145 (2004).

[63] S. Deser, R. Jackiw, and G. 't Hooft, Three-dimensional einstein gravity: Dynamics of flat space, Ann. Phys. (N.Y.) 152, 220 (1984).

[64] S. Deser and R. Jackiw, Three-dimensional cosmological gravity: Dynamics of constant curvature, Ann. Phys. (N.Y.) 153, 405 (1984).

[65] G. T. Horowitz and A. R. Steif, Singular string solutions with nonsingular initial data, Phys. Lett. B 258, 91 (1991).
[66] A. Giacomini, R. Troncoso, and S. Willison, Threedimensional supergravity reloaded, Classical Quantum Gravity 24, 2845 (2007).

[67] G. Barnich, L. Donnay, J. Matulich, and R. Troncoso, Super-BMS $\mathrm{BM}_{3}$ invariant boundary theory from threedimensional flat supergravity, J. High Energy Phys. 01 (2017) 029.

[68] B. Chen, J. Long, and Y.-N. Wang, Conical defects, black holes and higher spin (Super-)symmetry, J. High Energy Phys. 06 (2013) 025.

[69] Yu. M. Zinoviev, Hypergravity in $\mathrm{AdS}_{3}$, Phys. Lett. B 739, 106 (2014).

[70] M. Henneaux, A. Perez, D. Tempo, and R. Troncoso, Hypersymmetry bounds and three-dimensional higherspin black holes, J. High Energy Phys. 08 (2015) 021.

[71] The analysis of $(M, N)$-extended hypergravity on $\mathrm{AdS}_{3}$ and its asymptotic structure has also been carried out in [72].

[72] M. Henneaux, A. Perez, D. Tempo, and R. Troncoso, Extended anti-de Sitter Hypergravity in $2+1$ Dimensions and Hypersymmetry Bounds, in Proceedings, International Workshop on Higher Spin Gauge Theories: Singapore, Singapore, 2015 (World Scientific, Singapore, 2017), pp. 139-157.

[73] As in $D=3$, the hyper-Poincaré algebra can also be readily written in terms of Majorana spinors when they exist. In two spacetime dimensions the algebra is still consistent, but nevertheless, the subset spanned by translations and fermionic generators becomes an Abelian ideal.

[74] M. Banados, R. Troncoso, and J. Zanelli, Higher dimensional Chern-Simons supergravity, Phys. Rev. D 54, 2605 (1996).

[75] Note that in four-dimensional spacetimes the coupling of each of these fermionic fields with gravitation becomes inconsistent $[9,23]$. It is also worth pointing out that the fermionic action (30) appears to be devoid of an analog of the local shift symmetry $[25,26,76]$ that allows to consistently gauge away the spinorial two-form from the analysis.

[76] R. Rahman, Frame- and metric-like higher-spin fermions, Universe 4, 34 (2018).

[77] T. Curtright, Generalized gauge fields, Phys. Lett. 165B, 304 (1985).

[78] M. Henneaux, V. Lekeu, and A. Leonard, A note on the double dual graviton, J. Phys. A 53, 014002 (2020).

[79] V.E. Lopatin and M. A. Vasiliev, Free massless bosonic fields of arbitrary spin in $d$-dimensional de Sitter space, Mod. Phys. Lett. A 03, 257 (1988).

[80] K. Alkalaev, FV-type action for $\mathrm{AdS}_{5}$ mixed-symmetry fields, J. High Energy Phys. 03 (2011) 031.

[81] R. Haag, J. T. Lopuszanski, and M. Sohnius, All possible generators of supersymmetries of the s matrix, Nucl. Phys. B88, 257 (1975).

[82] M. Banados, L. J. Garay, and M. Henneaux, The local degrees of freedom of higher dimensional pure ChernSimons theories, Phys. Rev. D 53, R593 (1996).

[83] M. Banados, L. J. Garay, and M. Henneaux, The dynamical structure of higher dimensional Chern-Simons theory, Nucl. Phys. B476, 611 (1996). 
[84] O. Chandia, R. Troncoso, and J. Zanelli, Dynamical content of Chern-Simons supergravity, AIP Conf. Proc. 484, 231 (1999).

[85] O. Miskovic, R. Troncoso, and J. Zanelli, Canonical sectors of five-dimensional Chern-Simons theories, Phys. Lett. B 615, 277 (2005).

[86] O. Miskovic, R. Troncoso, and J. Zanelli, Dynamics and BPS states of $\operatorname{AdS}(5)$ supergravity with a Gauss-Bonnet term, Phys. Lett. B 637, 317 (2006).

[87] M. Hassaine, R. Troncoso, and J. Zanelli, Poincare invariant gravity with local supersymmetry as a gauge theory for the M-algebra, Phys. Lett. B 596, 132 (2004).

[88] M. Hassaine, R. Troncoso, and J. Zanelli, 11D supergravity as a gauge theory for the M-algebra, Proc. Sci., WC2004 (2005) 006 [arXiv:hep-th/0503220].

[89] M. Banados, C. Teitelboim, and J. Zanelli, Dimensionally continued black holes, Phys. Rev. D 49, 975 (1994).

[90] J. Crisostomo, R. Troncoso, and J. Zanelli, Black hole scan, Phys. Rev. D 62, 084013 (2000).

[91] E. Gravanis and S. Willison, 'Mass without mass' from thin shells in Gauss-Bonnet gravity, Phys. Rev. D 75, 084025 (2007).

[92] S. Deser and A. Waldron, Gauge Invariances and Phases of Massive Higher Spins in (A)dS, Phys. Rev. Lett. 87, 031601 (2001).

[93] S. Deser and A. Waldron, Partial masslessness of higher spins in (A)dS, Nucl. Phys. B607, 577 (2001).

[94] E. D. Skvortsov and M. A. Vasiliev, Geometric formulation for partially massless fields, Nucl. Phys. B756, 117 (2006).

[95] O. Fuentealba, J. Matulich, and R. Troncoso (to be published).
[96] N. L. González Albornoz, D. Lust, S. Salgado, and A. Schmidt-May, 4D spin-2 fields from 5D Chern-Simons theory, J. High Energy Phys. 12 (2018) 026.

[97] J. Engquist and O. Hohm, Higher-spin Chern-Simons theories in odd dimensions, Nucl. Phys. B786, 1 (2007).

[98] J. Engquist and O. Hohm, Higher-spin dynamics and Chern-Simons theories, Fortsch. Phys. 56, 895 (2008).

[99] R. Manvelyan, K. Mkrtchyan, R. Mkrtchyan, and S. Theisen, On higher spin symmetries in $\mathrm{AdS}_{5}$, J. High Energy Phys. 10 (2013) 185.

[100] An intriguing link between the hyper-Poincaré algebra with fermionic generators of half-integer spin and the extended $s$ conformal Galilean algebra in three dimensions, being purely bosonic, has been recently pointed out in [101] (see also [102]). Further aspects of the hyper-Poincaré algebra and its deformations have also recently been discussed in [103,104].

[101] D. Chernyavsky and D. Sorokin, Three-dimensional (higher-spin) gravities with extended Schrödinger and $l$ conformal Galilean symmetries, J. High Energy Phys. 07 (2019) 156.

[102] D. Chernyavsky, N. S. Deger, and D. Sorokin, Spontaneously broken $3 d$ hietarinta-maxwell chern-simons theory and minimal massive gravity, arXiv:2002.07592.

[103] S. Bansal and D. Sorokin, Can Chern-Simons or RaritaSchwinger be a Volkov-Akulov Goldstone? J. High Energy Phys. 07 (2018) 106.

[104] S. Bansal, Goldstone Fields with Spins Higher than 1/2, Ph.D. thesis, U. Padua, Dept. Phys. Astron., 2018.

[105] O. Fuentealba, J. Matulich, and R. Troncoso, Hypergravity in odd-dimensional spacetimes, Report No. CECS-PHY$19 / 01$. 